\title{
A CST Simulation and Performance Analysis for a Passive Façade integratable Metamaterials Structure
}

\author{
Tianhua Meng ${ }^{1,2^{*}}$, Giorgio Savini ${ }^{2}$, Christopher Leung ${ }^{4}$, Guozhong Zhao ${ }^{3}$, Fusheng Deng ${ }^{1}$
}

\author{
${ }^{1}$.Department of Physics and \\ Electronics Science \\ Shanxi Datong University \\ Datong, China
}

\author{
${ }^{2}$.Department of Physics and \\ Astronomy \\ University College London \\ London, UK
}

\author{
${ }^{3}$.Department of Physics \\ Capital Normal University \\ Beijing, China
}

\author{
${ }^{4}$.The Bartlett School of \\ Architecture \\ University College London \\ London,
}

\begin{abstract}
In the case of stone relics with cultural heritage value that are exposed to the adverse effects of direct exposure to weathering, we present a metamaterial with a composite structure that can passively control the transmittance of infrared and visible light. This periodic multi-layer structure consist of metal and dielectric layers, these have the function of low transmittance at infrared wavelengths and high transmittance in visible light. Its spectrally-selective behavior offers the possibility of both temperature regulation and natural daylighting of cultural relics. In addition, it can be discretely integrated with an existing architectural façade to provide weathering protection against rain and snow. Results show the composite structure can not only obtain a band gap width of $109 \mathrm{THz}$ in the near infrared wave-band but also ensure that most of the visible light's transmittance is higher than $\tau=0.4$. Furthermore, since the transmission spectrum is independent of polarization angle, the cultural relics benefit from its spectral-selective properties regardless of the polarization angle due to the sun's position.
\end{abstract}

Keywords-Simulation; Passive thermal-regulation; Selective light-transmission; Preservation of cultural heritage, stone relics

\section{INTRODUCTION}

Stone relics of high cultural value are often large, located outdoors and in environmental contexts that present challenges for their protection against the damaging effects of weathering. The study of materials and technology for conservation is an important topic in the field of international cultural heritage [13]. Many factors contribute to the deterioration of a stone relic when located outdoors, these include the effects of weathering, microbial activity and the chemical interaction with minerals in the stone if water ingresses [4-8]. Amongst these, the weathering effects from temperature fluctuations around outdoor stone relics are strongly dependent on exposure to the sun's visible and infrared light. Given that $95 \%$ of thermal radiation energy is in the near infrared (NIR) region between $\lambda=(720$ to 2500$) \mathrm{nm}$, we focus on designing the optical transmission and reflection properties of a metamaterial in this region.

Stone relics exposed to natural precipitation suffer the weathering effects of water ingress, this is known to lead to 978-1-5386-2720-4/17/\$31.00 @2017 IEEE damage from physical, chemical and biological processes [6]. A material that can be integrated within an existing architectural façade to stop the intrusion of precipitation would mitigate these effects. In addition, it would contribute to dampening the stone relic's exposure to diurnal fluctuations in the outdoor thermal environment. There are a range of different approaches to the preservation of cultural heritage, including protective methods and materials applied directly to stone relics [9-12]. In each case, whether by water proofing, acid or base protection, anti-fouling, corrosion or inhibiting the growth of microbes, there are numerous side-effects and these often only slow the process of deterioration and can in some cases even reinforce its effects.

Fewer studies have been undertaken to apply passive preventative measures to incorporate within the enclosures that surround relics as a method to mitigate weathering processes. This study is aimed at designing the optical properties of a material to passively differentiate the incident radiation between the relic's enclosure and its surroundings that most influence temperature fluctuations. While also forming a weather barrier that can be integrated into an existing façade to mitigate against water ingress.

This paper presents a new approach to weathering protection of stone relics with a passive spectrally-selective material by means of numerical simulation and theoretical analysis. A periodic multilayer structure that consists of metal and dielectric layer has been developed. It can control transmission of infrared and visible light [13-16]. The structure can be integrated within existing façade openings as it is extremely thin, transparent to the eye and would present a low profile for replacing existing or damaged windows. Not only would this structure provide weathering protection with minimal alteration to the existing cultural relics, it would play an important role in preserving culturally valuable relics. This also has practical significance for the research and development of new materials for slowing the deterioration of heritage relics by weathering processes.

Periodic multilayer structures have been designed, parameters optimized and its mechanisms analyzed using 
Computer Simulation Technology Microwave Studio (CST MWS) as described previously in [17-20]. The results showed that metamaterials can be effectively designed to have spectrally selectively behavior, in this case low transmittance in the infrared wavelength and high transmittance in visible light. Structural design and theory study

\section{A. Weathering Protection Structure}

The design of the metamaterial weathering protection structure is based on the simultaneous resonant excitation of an electric dipole and magnetic dipole. Computer simulations in CST were used to study and optimize the light transmission characteristic with respect to the structure's thickness. Our metamaterial weathering protection structure consists of a substrate upon which unit cells are deposited. Referring to Fig. 1 (a) each unit cell is a multi-layer consisting of insulatormetal-insulator-metal-insulator-metal-insulator-metal. These unit cells are then patterned into a two dimensional periodic array along $\mathrm{x}$ and $\mathrm{y}$ directions as shown in Fig. 1 (a). From the substrate layer upwards, each of the metal layers in a unit cell alternates between having a circular ring (C) and a hollow square block (B), this configuration was selected because it is expected to give rise to differences in the resonance excitation of incident light. Its resonance excitation properties are also independent of the light's polarization. The metal layers are of Aluminum (Al) which was selected due to its chemical stability and low Ohmic loss. The dielectric layers are of silica $\left(\mathrm{SiO}_{2}\right)$, selected for its excellent absorption in the infrared and desirable thermal and mechanical properties. The dimension of the $\mathrm{SiO}_{2}$ substrate and metal layers are specified in Fig. 1 (b).

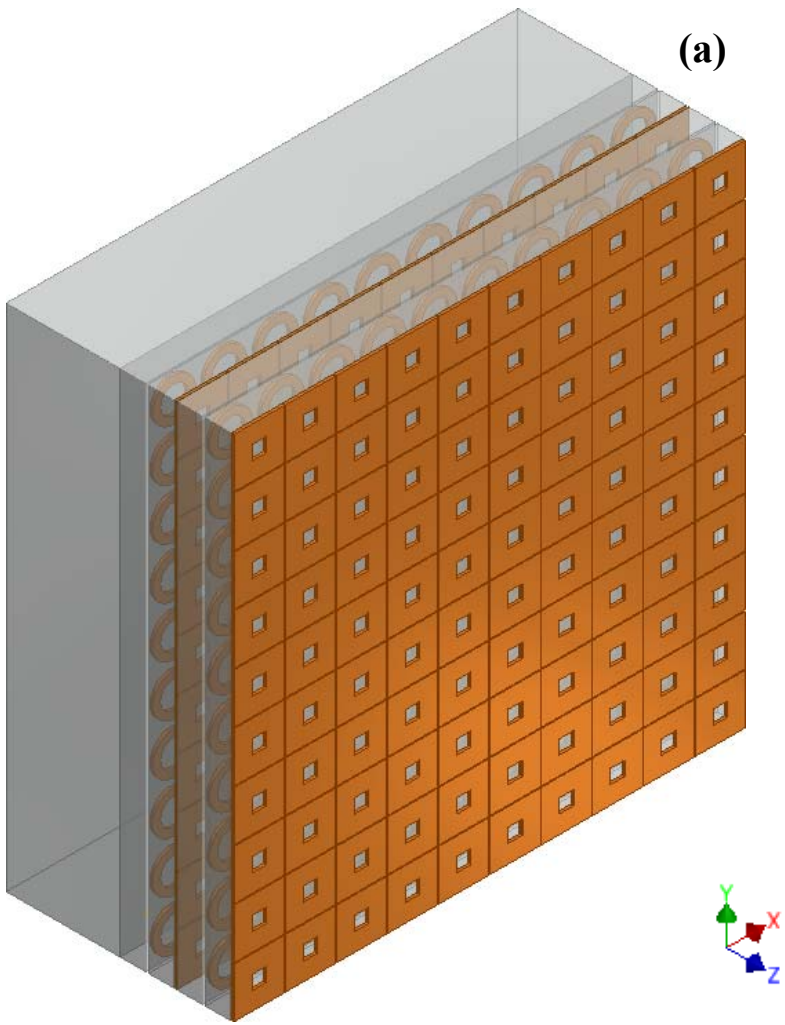

Fig. 1 (a) Schematic of the weathering protection structure with its periodic array of rings (C) and hollow blocks (B).

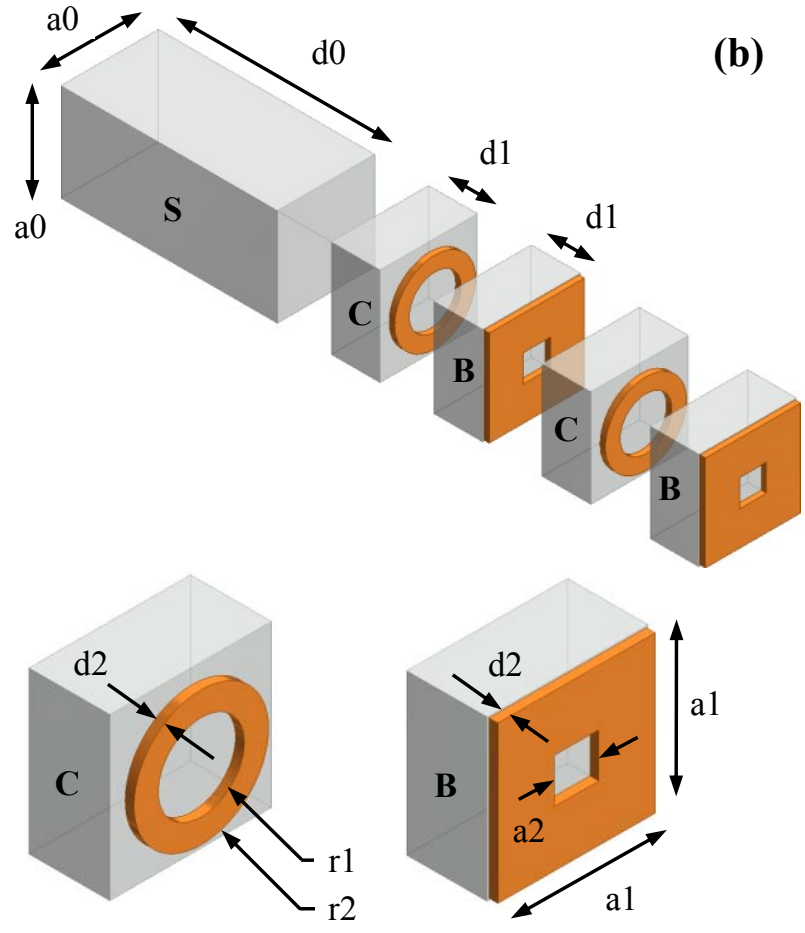

Fig. 1 (b) Exploded schematic showing unit cell structure: Consisting of two components: Cylindrical (C) and Block (B). The dimensional parameters are $\mathrm{d} 0=200 \mathrm{~nm}, \mathrm{~d} 1=45 \mathrm{~nm}, \mathrm{~d} 2=5 \mathrm{~nm}, \mathrm{a} 0=90 \mathrm{~nm}, \mathrm{a} 1=88 \mathrm{~nm}, \mathrm{a} 2=25 \mathrm{~nm}, \mathrm{r} 1=37.5$ $\mathrm{nm}, \mathrm{r} 2=24 \mathrm{~nm}$.

\section{B. Parameter Settings}

The properties of the weathering protection structure were calculated using the CST electromagnetic simulation software based on the finite element method. The interaction between incident radiation and the sub-wavelength geometry of the metal Al layer on a dielectric substrate leads to plasma excitation. This is due to the combination of electrical conductivity and the equivalent dielectric constant in both the metal and the dielectric.

The dielectric permittivity of $\mathrm{Al}$ was modeled using the Drude expression:

$$
E(\omega)=1-\frac{\omega_{p}^{2}}{\omega(\omega+\mathrm{i} \gamma)}
$$

With the plasma frequency $\omega_{\mathrm{p}} / 2 \pi=3.664 \cdot 10^{15}$ and damping frequency $\gamma / 2 \pi=1.941 \cdot 10^{13}$, in the infrared wavelengths of interest here the equivalent dielectric permittivity of $\mathrm{Al}$ is taken as $\gamma=5.1$ and the dielectric permittivity of $\mathrm{SiO}_{2}$ is taken as $\gamma=2.25$ based on well-known experimental results in [20].

Given metamaterial layers in the $\mathrm{X}-\mathrm{Y}$ plane, radiation is assumed to be incident along the $\mathrm{Z}$-axis. The three dimensional unit cell was simulated using periodic boundary conditions along the $\mathrm{X}-\mathrm{Y}$ directions so that structure can be regarded as an infinite two-dimensional array. The incident radiation is a transverse electromagnetic wave applied using wave-port boundary conditions. The frequency dependent transmittance (T) was obtained from the S-parameters in the simulation package. The frequency range set as follows: $100 \sim 800 \mathrm{THz}$, and the solver settings accuracy set at $-60 \mathrm{~dB}$. 


\section{RESULTS AND DISCUSSION}

\section{A. Simulation results}

In order to assess the most suitable weathering protection structure, the transmission spectra of different configurations where evaluated. These include: a single cylinder, a single square, combinations of the cylinder and the square structures. In each case, the effects of vertical light irradiation on the structure were simulated. Fig 2 shows the transmission spectra for all of the structures. The results showed that the single square structure (B) has a distinct trough at $291.1 \mathrm{THz}$, the single cylindrical structure (C) appeared at $366 \mathrm{THz}$, the combination of the block and cylindrical structure $(\mathrm{B}+\mathrm{C})$ has a transmission band gap in the range of (291.1 to 325.4) THz. c (250 to 359$) \mathrm{THz}$, which covers most of the NIR region taken as (120 to 417$) \mathrm{THz}$. The change of the band gap was minimal after adding the substrate (S) this showed a small improvement in transmission at low frequencies.

The low transmittance of this structure in the NIR matches the long wavelength radiation that is re-emitted from the stone relics within the grotto itself. This property mitigates against the risk of damage from thermal heating by dampening temperature fluctuations. It also maintains high transmission in visible light allowing tourists and visitors unimpeded visibility. In addition, the weathering protection structure with substrate are easier to combine with the original eaves than the structure without substrate.

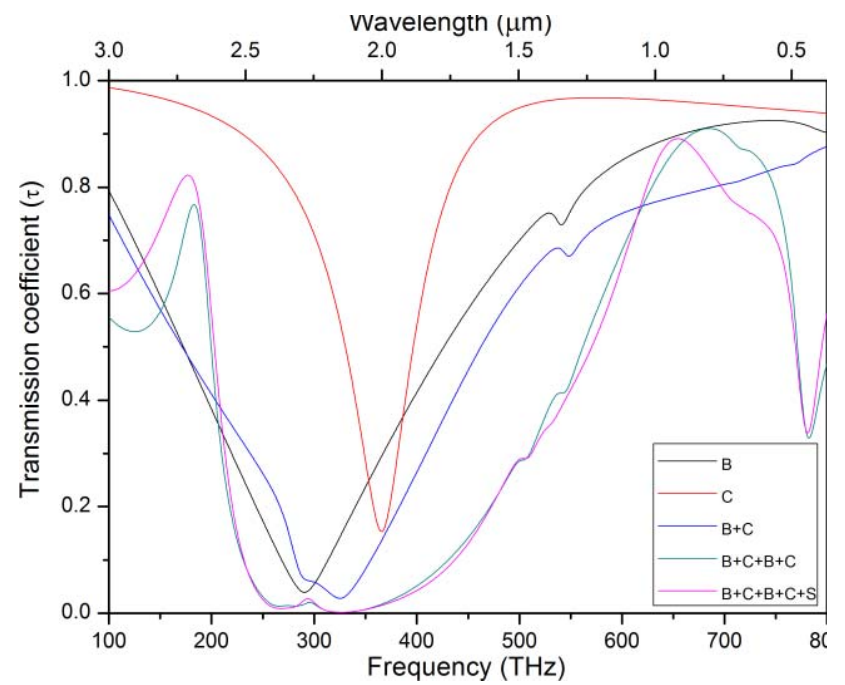

Fig. 2. The transmission spectra all of the structures

In the context of a real-world application, the direct-beam from sunlight under a clear sky is incident from a range of angles depending upon the time of day. To consider the structure's transmissivity under these conditions and account for sunlight's polarization, a range of angles of incidence were simulated and its influence on transmissivity analyzed. Figure 3 shows the results of the simulation under these conditions. Overall, the results show that the transmission coefficient is largely independent of the angle of incidence. However, the width of the band gap does narrow with a lower transmittance at both small and large angles of incidence within $10^{\circ}$ of the extremes. Therefore in practical applications, the structure's beneficial properties can be exploited irrespective of solar position, without adverse effects on statues or relics.

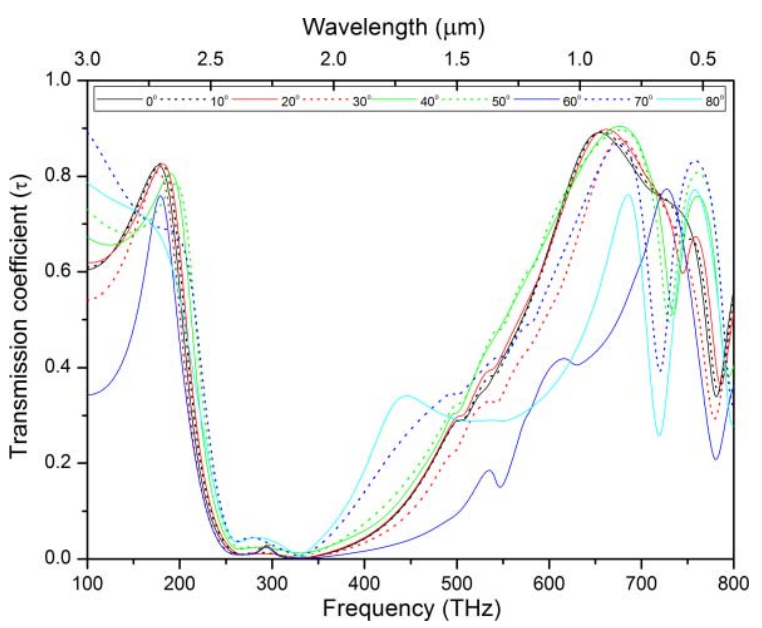

Fig. 3. The transmission spectrum of the weathering protection structure for different angles of incidences

\section{B. Mechanism analysis}

In order to study the electronic vibration mode of the surface plasma of the weathering protective structure, we explored the electromagnetic properties, which represents the near field intensity of electric field distribution at the wave trough for each configuration of unit cell structure by setting up a Field Distribution Monitor as shown in (Fig 4, 5).

First, the distribution of electric field in the trough (291.1 $\mathrm{THz}$ ) of the single square structure (B) was calculated, as shown in Fig 4. The electric field energy is limited to the outer edge of $y$ direction square ring, which means the plasma resonance trough arises from the dipole vibration mode of the electrons in the vertical direction. While the distribution of electric field in the trough $(366 \mathrm{THz})$ of the single cylindrical structure $(\mathrm{C})$ indicates that the electric field energy is limited to the middle of the inner ring, which creates a trough of transmission spectrum here (Fig 4).

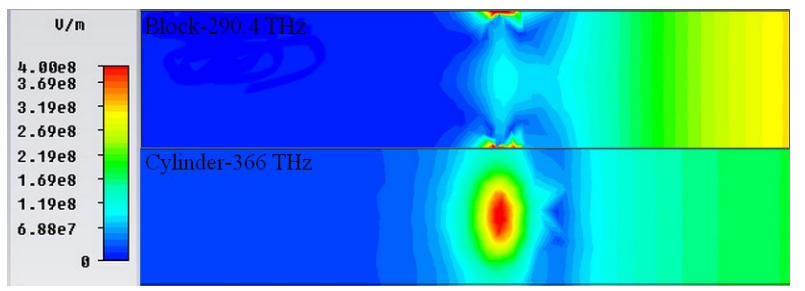

Fig. 4. The distribution of electric field at the trough of the single structures

In addition, we also simulated the electric field intensity distribution of the combination structure at the two divided troughs of the forbidden band boundary (Fig 5). We show the complex electromagnetic properties of combining rectangular and cylindrical composite structures, with the electric field energy mainly localized in the square ring structure at low frequency $(291.1 \mathrm{THz})$ while localized in the round cylinder structure at high frequency $(325.4 \mathrm{THz})$. It suggests that the electromagnetic performance of the combined structure originates from the separate structure, which has a stronger 
local surface electric field in the XY plane, corresponding to the band gap widening and the transmissivity decreasing between two troughs, especially the transmission coefficient at $325.4 \mathrm{THz}$ showed 0.03 down from 0.15 . In addition, the transmission spectrum of the combination structure had no obvious change after adding the substrate. Therefore, the combination of two rectangular and cylindrical and substrate structure is believed to be suitable for the cell style of structure, which can be used for weathering protection of the outdoor stone relics.

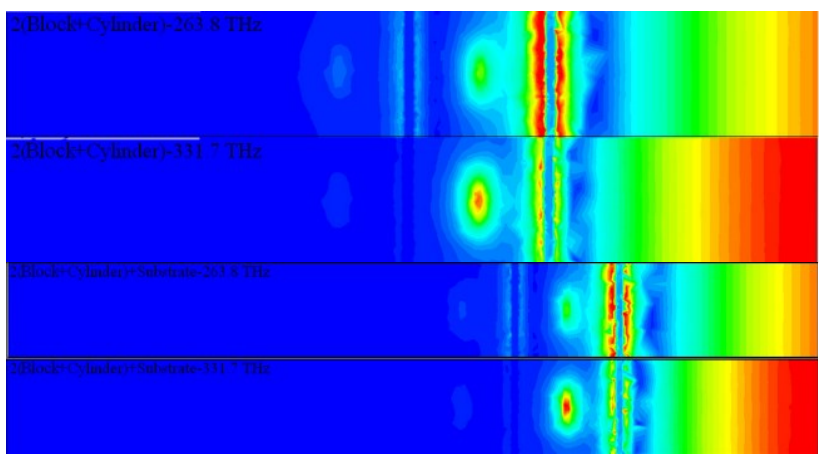

Fig. 5. The distribution of electric field at the trough of the combination structures

\section{CONCLUSION}

In this paper, weathering protection structure was designed and modelled with CST simulation software. The transmission spectra of the protective structure unit and each composite structure were computed, and the electric field intensity distribution corresponding to transmission troughs were studied. The results showed that the alternative combination of the block and cylindrical and the substrate structure has a wider transmission band gap in the NIR band, however it can ensure high transmission at visible wavelength. Moreover, the resonance excitation properties are also independent of the light's polarization. Therefore, the protection structure has practical significance for the research and development of new method for slowing the deterioration of heritage relics by weathering processes.

\section{ACKNOWLEDGMENT}

The National Natural Science Foundation of China under Grant No 11504212, F050408, and 11274207, the Key Industrial Science and Technology Projects of Datong under Grant No 2015015 and 2015016, and the PhD research startup foundation of Shanxi Datong University under Grant No 2014B06 supported this research.

\section{REFERENCES}

[1] S Folarin, "International Standards for Cultural Heritage: An African PersPective," Art Antiquity and Law, vol. 5, pp. 159-175, Feb. 2000.
[2] G Graziani, E Sassoni, and E Franzoni, "Consolidation of porous carbonate stones by an innovative phosphate treatment: mechanical strengthening and physical microstructural compatibility in comparison with TEOS-based treatments," Heritage Science, vol 3, pp. 1-6, Jan. 2015 .

[3] A Chabas, and D Jeannette, "Weathering of marbles and granites in marine environrnent: Petrophysical properties and special role of atmospheric salts," Environmental Geology, vol. 40, pp. 359-368, Jan. 2001 .

[4] P Brimblecombe, and P Lankester, "Long-term changes in climate and insect damage in historic house," Studies in Conservation, vol. 58, pp. 13-22, Jan. 2013.

[5] E Sassoni, E Franzoni, B Pigino, G.W. Scherer, and S Naidu, "Consolidation of calcareous and siliceous sandstones by hydroxyapatite: comparison with a TEOS-based consolidant," J Cult Herit, vol. 14, pp. 103-111, Jun. 2013

[6] R.Z. Liu, B.J. Zhang, H Zhang, M.F. Shi, "Deterioration of Yungang Grottoes: Diagnosis and research," J Cult Herit, vol. 12, pp. 494-499, Oct. 2011

[7] F Yan, Q.Y. Ge , Q Li, M Yu, X.D. Zhu, and J Pan, "Analysis of microbial community on the surface of the historic stone and nearby rock samples in Yungang Grottoes," Acta Microbiol. Sin, vol. 52, pp. 629-636, May. 2012

[8] A.P. Ferreira Pinto, and R.J. Delgado, "Consolidation of carbonate stones: influence of treating procedures on the strengthening action of consolidants," J Cult Herit, vol. 13, pp. 154-166, Jan. 2012

[9] D.C.Creagh, "The characterization of art effects of cultural heritage signicance using physical techniques," Radiation Physics and Chemistry, vol. 74, pp. 426-442, Dec. 2005.

[10] D.M. Han, and G.S. Guo, "The Application of the chemica consolidation in conservation of stone," J. Sci Conserv Archaeol, vol 11, pp. 41-43, Jan. 1999.

[11] P Cardiano, R.C. Ponierio, S Sergi, and S.L. Sehiavo, "Epoxy-Siliea Polymers as Stone Conservation Materials," Polymer, vol. 46, pp. 1857 1864, Feb. 2005

[12] S Enrico, and F Elisa, "Influence of porosity on artificial deterioration of marble and limestone by heating," Applied Physics A, vol. 115, pp 809-816, Jun. 2014.

[13] J.B. Pendry, D Schurig, and D.R.Smith, "Controlling electromagnetic fields," Science, vol. 312, pp. 1780-1785, Jun. 2006.

[14] M Tlidi, K Staliunas, K Panajotov, A.G. Vladimirov, and M.G. Clerc, "Localized Structures in Dissipative Media: From Optics to Plant Ecology. Philosophical Transactions of the Royal Society A: Mathematical," Physical and Engineering Sciences, vol. 372, pp. 1-18, Aug. 2014.

[15] L.Z. Lu, J.J. Wang, and Y.T. Fang, "Oblique total transmission through an anisotropic zero-epsilon metamaterial slab," Optics \& Laser Technology, vol. 47, pp. 4-9, Apri. 2013.

[16] J.B. Masson, and G Gallot, "Coupling between surface plasmons in subwavelength hole arrays," Physical Review B, vol. 73, pp. 121401(14), 2006.

[17] W.L. Barnes, A Dereux, and T.W. Ebbesen, "Surface plasmon subwavelength optics," Nature, vol. 424, pp. 824-830, March. 2003

[18] S.G Liu, P Zhang, and W.H. Liu, "Surface Polariton Cherenkov Light Radiation Source,” Phys. Rev. Lett., vol. 109, pp. 153902-153906, Oct. 2012.

[19] F Miyamaru, and M Hangyo, "Strong optical activity in chiral metamaterials of metal screw hole arrays," Applied Physics Letters, vol. 89 , pp. 211105-211108, Nov. 2006.

[20] G Dayal, and S. Anantha Ramakrishna, "Design of highly absorbing metamaterials for Infrared frequencies," Optics Express, vol. 20, pp 16(1-6), July. 2012. 